\title{
Propose Data Mining AR-GA Model to Advance Crime analysis
}

\author{
Emad K. Jabar ${ }^{1}$, Soukaena H. Hashem ${ }^{1}$, Enas M. Hessian ${ }^{2}$ \\ ${ }^{1}$ Computer Sciences/ University of Technology/ Baghdad-Iraq \\ ${ }^{2}$ Computer Sciences/ Al-Mustansaria University / Baghdad-Iraq
}

\begin{abstract}
Historically solving crimes has been the privilege of the criminal justice and law enforcement specialists. With the increasing use of the computerized systems to track crimes, computer data analysts have started helping the law enforcement officers and detectives to speed up the process of solving crimes. According to, solving crimes is a complex task that requires human intelligence and experience. In this research we belief data mining is a technique that can assist law enforcement officers with crime detection problems, so the proposal tries to benefits years of human experience into computer models via data mining. Here we will take an interdisciplinary approach between computer science and criminal justice to develop a proposed data mining model. The proposed model is a three correlated dimensional model; each dimension is a datasets, first one present crime dataset second present criminal dataset and the third present geo-crime dataset. This model apply the Association Rules AR data mining algorithm on each of the three correlated dataset separately then using Genetic Algorithm GA as mixer of the resulted ARs to exploit the relational patterns among crime, criminal and geo-crime to help to detect universal crimes patterns and speed up the process of solving crime with more accurate. This research introduces suggestion to secure the results of the data mining association rules. For privacy preserving secure datasets we aim to hide the general secure and sensitive rules from appearing as a result of applying AR. This could be done by making the confidence of secure rules equal to zero by modifying the supports of critical and sensitive items in these rules. The proposal applied on real crime data from a dependable sheriff's office and validated our results.
\end{abstract}

Keywords: Crime Analysis, Data Mining, AR, GA, Crimnal.

\section{Introduction}

Crime is a major issue where the top priority has given by all government. The data for crime often presents an interesting dilemma. While some data is kept confidential, some becomes public information. Data about the prisoners can often be viewed in the county or sheriff's sites. However, data about crimes related to narcotics or juvenile cases is usually more restricted. Similarly, the information about the sex offenders is made public to warn others in the area, but the identity of the victim is often prevented. Thus as the analyst has to deal with all these public versus private data issues so that analysis modeling process does not infringe on these legal boundaries. Law enforcement agencies like that of police today are faced with large volume of data that must be processed and transformed into useful information. The high volume of crime datasets and also the complexity of relationships between these kinds of data have made criminology an appropriate field for applying data mining techniques. Identifying crime characteristics is the first step for developing further analysis. The knowledge that is gained from data mining approaches is a very useful tool which can help and support police force. It is one of the most important fields where the application of data mining techniques can produce important results $[1,2]$.

Criminology present area that focuses the scientific study of crime and criminal behavior and law enforcement and is a process that aims to identify crime characteristics. Crime analysis is a task that includes exploring and detecting crimes and their relationships with criminals. Crime analysis is a set of systematic, analytical processes directed at providing timely and pertinent information relative to crime patterns and trend correlations to assist operational and administrative personnel in planning the deployment of resources for the prevention and suppression of criminal activities, known as crime analysis [3].

Crime analysis need data mining, because the last is an iterative process of extracting knowledge hidden from large volumes of raw data. The knowledge must be new, not obvious, and one must be able to use it. It is a tool to extract diamonds of knowledge from historical data and predict outcomes of future situations. Data mining involves the use of sophisticated data analysis tools to discover previously unknown, valid patterns and relationships in large data sets. Consequently, data mining consists of more than collecting and managing data, it also includes analysis and prediction. The main reasons to use data mining with crime analysis are: too much data but too little information, and a need to extract useful information from the data and to interpret the data. The challenge in data mining crime data often comes from the free text field. While free text fields can give the newspaper columnist, a great story line, converting them into data mining attributes is not always an easy job $[4,5]$. 


\section{Related Works}

In [6] Malathi. A. et. al., they discuss that, a major challenge facing all law-enforcement and intelligence gathering organizations is accurately and efficiently analyzing the growing volumes of crime data. There has been an enormous increase in the crime in the recent past. they look at MV algorithm, DB Scan and PAM outlier detection algorithm with some enhancements to aid in the process of filling the missing value and identification of crime patterns. They applied these techniques to real crime data and use semi-supervised learning technique here for knowledge discovery from the crime records and to help increase the predictive accuracy. In [7] Mande U. et. al., introduce binary clustering and classification techniques have been used to analyze the criminal data. The crime data considered in this paper is from Andhra Pradesh police department this paper aims to potentially identify a criminal based on the witness/clue at the crime spot an auto correlation model is further used to ratify the criminal. In [8] Malathi. $\boldsymbol{A}$ et. al., they use a clustering/classify based model to anticipate crime trends. The data mining techniques are used to analyze the city crime data from Police Department. The results of this data mining could potentially be used to lessen and even prevent crime for the forth coming years. In [9] Sathyaraj S. R. et. al., they studies to integrate a large volume of data sets into useful information by adopting a various information techniques in the hottest technology world. The adopted approaches of Single variate Association Rule for Crime to Crime based on the knowledge discovery techniques such as, clustering and association-rule mining. It reveals with an inherent of patterns of information into a fruitful exploratory tool for the discovery of spatio-temporal patterns. This tool is an autonomous pattern detector to reveal plausible cause-effect associations between layers of point and area data. Also present REPA, VBEA and TEA algorithms with an exploratory analysis for the effectively explore geo-referenced data. The present study of this paper was focuses through the real crime dataset by using various algorithms. In [10] Mande $\boldsymbol{U}$. et. al., they aims towards the construction of new methodologies based on Data mining concepts and serves as a decision support system. Given a set of available clues, from the forensic labs and the clues collected at the crime spot, a methodology is presented to map the evidence and identify a criminal. In [11] Chen N. et. al., their model, firstly, we predict the residence of the offender based on the locations of the last crime scenes with three methods (distance analysis: the location that has the shortest distance to each crime site, circle fitting, probability theory); secondly, they predict the time of the next crime based on previous data with the method of fitting a straight line; next, predict the location of the next crime based on the locations of the last crime scenes and the time predicted in the second step with the method of weighted average; finally, generate a predicted location based on the three predicted locations with the method of weighted average. In [12] Yu C. H. et. al., they discuss the preliminary results of a crime forecasting model developed in collaboration with the police department of a United States city in the Northeast. They first discuss approach to architecting datasets from original crime records. The datasets contain aggregated counts of crime and crime-related events categorized by the police department. The location and time of these events is embedded in the data. Additional spatial and temporal features are harvested from the raw data set. Second, an ensemble of data mining classification techniques is employed to perform the crime forecasting. In [13] Al-Janabi K. B. S., they presents a proposed framework for the crime and criminal data analysis and detection using Decision tree Algorithms for data classification and Simple K Means algorithm for data clustering. The paper tends to help specialists in discovering patterns and trends, making forecasts, finding relationships and possible explanations, mapping criminal networks and identifying possible suspects. The classification is based mainly on grouping the crimes according to the type, location, time and other attributes; Clustering is based on finding relationships between different Crime and Criminal attributes having some previously unknown common characteristics.

\section{The Proposal of Crime Analysis}

To present the proposed three correlated dimensions model of crime analysis and prediction using data mining, first will introduce a big view of this model explained in the following algorithm, see figure (1).

\section{General Algorithm of Proposal Model}

Input : Raw of crime data from depended sheriff's office.

Output: Three correlated dimensions model for crime analysis and prediction

Steps:

1. Understanding the crime domain, this includes relevant prior knowledge and goals of the crime detection and prediction.

2. Extracting the target dataset, this is for building a dataset for the three dimensions of the proposed model; crime, criminal and geo-crime. Or focusing on a subset of variables, in our proposal feature selection will done by taking all features could not affected by criminal surgery, crime conflictions and geo-crime environment changes. 
3. Data preprocessing, this is required to improve the quality of the actual data for mining. This also increases the crime mining efficiency by reducing the time required for mining the preprocessed data. In our proposal we concentrate on data preprocessing to involve data cleaning and treating missing values.

4. Data mining, data mining AR applied on each dimension dataset separately then mixing the resulted AR by GA to introduce correlated patterns among the three dimensions to advance the crime analysis.

5. Interpretation and Using discovered knowledge, this includes interpreting the discovered patterns, by providing SQL on-demand or reports for both separated dimension and correlated dimensions. Using knowledge it includes incorporating this knowledge into the performance system and taking actions based on the knowledge.

6. end

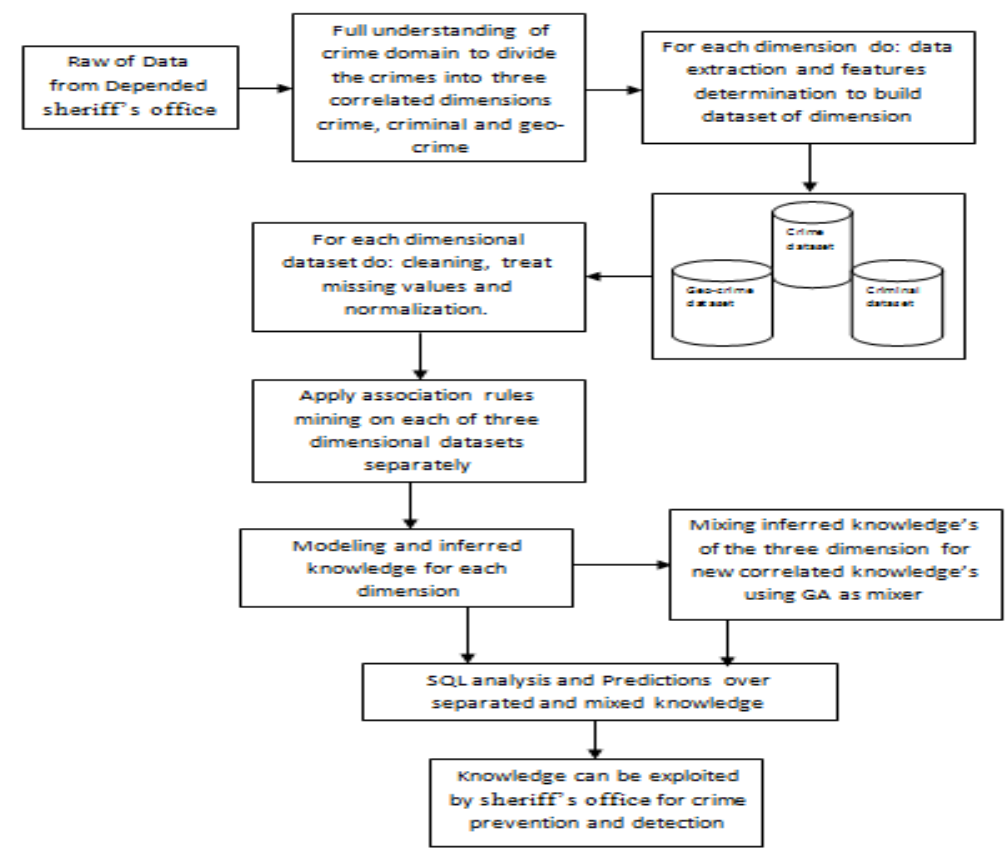

Figure (1): a proposed three correlated dimensional data mining model.

\subsection{Understanding the Crime Domain}

Crime is neither systematic nor entirely random, so crime analysis tool should be able to identify crime patterns quickly and in an efficient manner for future crime pattern detection. The major challenges are encountered.

- Crime information volume has been increased.

- Different methods and structures used for recording crime data.

- The data available is inconsistent and are incomplete thus making the task of formal analysis a far more difficult.

The main focus is to develop a crime analysis tool that assists the police in:

- To perform crime analysis to detect crime patterns.

- Provide information to formulate strategies for crime prevention and reduction.

- Identify and analyze common crime patterns to reduce further occurrences of similar incidence.

Now will present our objectives in adopting data mining in crime analysis and challenges will faces the crime analysis when use data mining.

Crime rates are rapidly changing and best analysis using data mining enables discerning hidden patterns of crime, if any, without any explicit prior knowledge of these patterns. The proposal has main objectives of crime analysis can be classified into:

1. Extraction of crime patterns by analysis of available correlated crime, criminal and geo-crime data.

2. Prediction of a crime based on the correlation of existing data and anticipation of crime rate using data mining techniques.

Data mining systems rely on databases to supply the raw data for input and this raises problems in that databases tend be dynamic, incomplete, noisy, and large. Other problems arise as a result of the adequacy and relevance of the information stored.

1. Limited Information, Inconclusive data causes problems because if some attributes essential to knowledge about the application domain are not present in the data it may be impossible to discover significant knowledge about a given domain. 
2. Noise and Missing Values, Databases are usually contaminated by errors so it cannot be assumed that the data they contain is entirely correct. Attributes which rely on subjective or measurement judgments can give rise to errors such that some examples may even be mis-classified. Errors in either the values of attributes or class information are known as noise.

3. Uncertainty, Uncertainty refers to the severity of the error and the degree of noise in the data. Data precision is an important consideration in a discovery system.

4. Size, Updates, and Irrelevant Fields, Databases tend to be large and dynamic in that their contents are ever-changing as information is added, modified or removed. The problem with this from the data mining perspective is how to ensure that the rules are up-to-date and consistent with the most current information. Also the learning system has to be time-sensitive as some data values vary over time and the discovery system is affected by the "timeliness" of the data.

5. Another issue is the relevance or irrelevance of the fields in the database to the current focus of discovery for example post-codes are fundamental to any studies trying to establish a geographical connection to an item of interest such as the sales of a product.

\subsection{Extracting the Target Dataset}

Here we see any crime investigation highlights primarily on three dimensions; these are crime dimension, criminal dimension and crime-geo dimension. For each dimension we select the most critical attributes (variables) are very interested and repeated in crime registration. Each dimension is a dataset has it is own attributes but all of the three datasets are correlated such that each transaction in them are related to one crime considering it is dimensions. Now will display the considered attribute in each dataset of dimensions:

Crime dataset take the following attributes (crime_id, crime_type, crime_location, crime_date, crime_weapon, crime_victum, crime_witness, crime_clues).

Criminal dataset take the following attributes (criminal_id, criminal_gender, criminal_age, criminal_address, criminal_income, criminal_job, criminal_maritalstatuse, criminal_signs_diffrence, criminal_- religon, criminal natioal)

Crime-geo dataset take the following attributes (geo-id, geo-population (high, medium, small), geo-size (large, medium, small), geo-type (town, river, forest,...), geo-earth (agriculture, industrial), geo_longitude, geo_latitude).

\subsection{Data Preprocessing}

Most of the data collection techniques for crime findings produce huge amount of information, where missing values, inconsistence data and unuseful data are inevitable. A data preprocessing is a process that consists of data cleaning, data integration and data transformation which is usually processed by a computer program. It intends to reduce some noises, incomplete and inconsistent data. The results from preprocessing step can be later proceeding by data mining algorithm. The preprocessing our models includes the following tasks:

Data cleaning: fill in missing values, smooth noisy data, identify or remove outliers, and resolve inconsistencies (there are many modest proposals for filling missing values).

Different preprocessing techniques were used to get clean data, these include:

- Removing outliers, some of the data in the crime, criminal and geo-crime datasets represent outliers and cannot be included in the analysis algorithms and techniques, so these data records were deleted from the set.

- Filling missing data, some criminal ages, jobs, and income were not mentioned in the tables, average and most commonly used values were used to substitute these missing values.

- Using KNN-based imputation method, in this method, the missing values of an instance are imputed by considering a given number of instances that are most similar to the instance of interest. The similarity of two instances is determined using a Euclidian distance function.

Data integration: using multiple databases, data cubes, or files (since our data are collected from many of sheriff's office so, the data are integrated to build uniform three datasets. That by uniform the selected attributes and uniforms the types of value's attributes.).

Data transformation: normalization and aggregation (in our model where the AR is the used technique for mining the attributes are all converted to letters appear if agree with attribute condition and disappear if not agree).

Data reduction: reducing the volume but producing the same or similar analytical results (in our model reducing done in two faces these are: face of omit some unusefull attributes such as criminal color eyes which the criminal could change it. The second face is omitting entire records because they have more than three missing values so the filling will cause noisy.). 
Data discretization: part of data reduction, replacing numerical attributes with nominal ones (in our model for example the attribute of age instead of filling it as number will be replaced by letter such as A, where A will appear if criminal age more than 35 year and disappear if age smaller).

\subsection{Data Mining Association Rules-Genetic Algorithm (AR-GA)}

The efficient discovery of such crime, criminal and geo-crime rules has been a major focus in our proposed data mining. Many algorithms and approaches have been proposed to deal with the discovery of different types of association rules discovered from a variety of databases. However, the proposed datasets relied upon are alphanumerical and often transaction-based. The problem of discovering association rules is to find relationships between the existence of an crime-attribute (or characteristic) and the existence of other crime-attributes (or characteristics) in a large repetitive collection.

The problem is stated as follows for each dimension, Let $I=\{i 1, i 2, \ldots i m\}$ be a set of literals, called items. Let $D$ be a set of transactions (dataset of dimension), where each transaction $T$ is a set of items such that $T$ $\subseteq I$. A unique identifier $T I D$ is given to each transaction. A transaction $T$ is said to contain $X$, a set of items in $I$, if $X \subseteq T$. An association rule is an implication of the form " $X \Rightarrow Y$ ", where $X \subseteq I, Y \subseteq I$, and $X \cap Y=\varnothing$. The rule $X \Rightarrow Y$ has a support $s$ in the transaction set $D$ is $s \%$ of the transactions in $D$ contain $X \cup Y$. In other words, the support of the rule is the probability that $X$ and $Y$ hold together among all the possible presented cases. It is said that the rule $X \Rightarrow Y$ holds in the transaction set $D$ with confidence $c$ if $c \%$ of transactions in $D$ that contain $X$ also contain $Y$. In other words, the confidence of the rule is the conditional probability that the consequent $Y$ is true under the condition of the antecedent $X$. The problem of discovering all association rules from a set of transactions $D$ consists of generating the rules that have a support and confidence greater than given thresholds. These rules are called strong rules.

Then after finding all association rules from the three datasets separately will mixing these rules to extract new rules to correlate these three datasets using genetic algorithm. Genetic algorithm will be applied on all association rules resulted from the three datasets as in the following proposed steps:

1. A genetic representation or encoding schema for potential solutions to the problem. Each association rule will be presented as a series of numbers (all alphabets representing the attributes will be encoded by numbers each number has two digit since we have many attributes distributed over all the three datasets. Such as $\mathrm{A}=01, \mathrm{~B}=02$, and finally the symbol ---> will take the number 00 ). For example the association rule from the third dataset is UVW---> XZ has the following encoding (212223002426).

2. One way to create an initial population of potential solutions, the initial population already created with association rules algorithms which established on the three datasets. So this means the initial population of GA will be all the association rules extracted by the three datasets separately and encoded as series of numbers.

3. An evaluation function that pays the role of the problem environment (novel association rules), rating solutions in term of their "fitness", Here the proposed evaluation function for each rule is consist of three parts, these are:

- Each number in each series is a two digit.

- Each number will appear only on the left or right of zero.

- Confidence of the rule must pass the minimum confidence.

4. Genetic operators that alter composition of offspring. One-point crossover is the most basic crossover operator, where a crossover point on the genetic code is selected at the zero number which occur in the series of numbers, and two parents rules are interchanged at this point.

5. Crossover exploits existing rule potentials, but if the population does not contain all the encoded information needed to find the novel rules, no amount of rules mixing can produce satisfactory solution. For this reason a mutation operator capable of spontaneously generating new frame is included. The most common way of implementing mutation is to flip a bit with a probability equal to a very low, given Mutation Rate (MR). A mutation operator can prevent any single bit from converging to a value through the entire population and, more important, it can prevent the population from converging and stagnating at any local optima.

6. Values for the various parameters that used by the genetic algorithm (population size, rate of applied operators, etc.). For our particular problem we use the following parameters of the genetic algorithm: population size (pop-size) $=3000$ (the parameter was already used) for each dataset will take the 1000 higher confidence rules. Probability of crossover $(\mathrm{PC})=1$, probability of mutation $(\mathrm{PM})=0.001$ (the parameter will be used in mutation operation).

7. Continue with genetic processing until the optimized rules will be optimized to be the novel rules. 


\subsection{Interpretation and Using discovered knowledge}

Visualization is an important aid that increases understandability from the perspective of humans. One can evaluate the mined patterns automatically or semi automatically to identify the truly interesting or useful patterns for the user. Given huge volumes of heterogeneous data, the objective is to efficiently extract meaningful patterns that can be of interest and hence useful to the user. The role of interestingness is to threshold or filters the large number of discovered patterns, and reports only those which may be of some use. The subjective approach, on the other hand, depends additionally on the user-who examines the pattern. Two major reasons why a pattern is interesting from the subjective (user-oriented) point of view are as follows. Unexpectedness: When it is "surprising" to the user and this potentially delivers new information to the user. Actionability: When the user can act on it to her/his advantage to fulfill the goal.

\subsection{Privacy preserving for Association Rules} following:

The proposed solution is to hide sensitive rules by reducing the confidence of these rules. Suppose the

- The rule is $\mathrm{A} \rightarrow \mathrm{B}$.

- $\quad$ Confidence $(\mathrm{A} \rightarrow \mathrm{B})=\operatorname{support}(\mathrm{AB}) / \operatorname{support}(\mathrm{A})$.

- Decreasing confidence of the rule by increasing the support of A in transactions without supporting B. Decreasing the support of B in transactions that supporting both A and B.

- Then surly the new Confidence of the rule $(\mathrm{A} \rightarrow \mathrm{B})=$ zero.

\section{Discussion and Results Experimental works}

The proposal had been implemented on the following platform: Windows 7 Ultimate Service Pack1 and 32-bit OS, 4GB RAM, and Intel ${ }^{\circledR}$ Core (TM) 2 Duo CPU with 2.00GHz; and by using Visual Basic 6.0 and SQL server. To prove our idea of building proposed three correlated dimensional model for advancing crime analysis and prediction we do the following Experimental works. Collecting data crimes from three sheriff's office, these data was collected from the year 2003 to year 2013. These data are divided in to two parts these parts are: First part was from 2003 to 2010, this part conducted to construct our proposed model. Second part was from 2010 to 2013 , this part conducted to verify the analysis and predictions discovered from applying proposed model on the first part data. On the first part apply data mining with GA for identifying crime patterns using the association rule techniques, see figures (2).

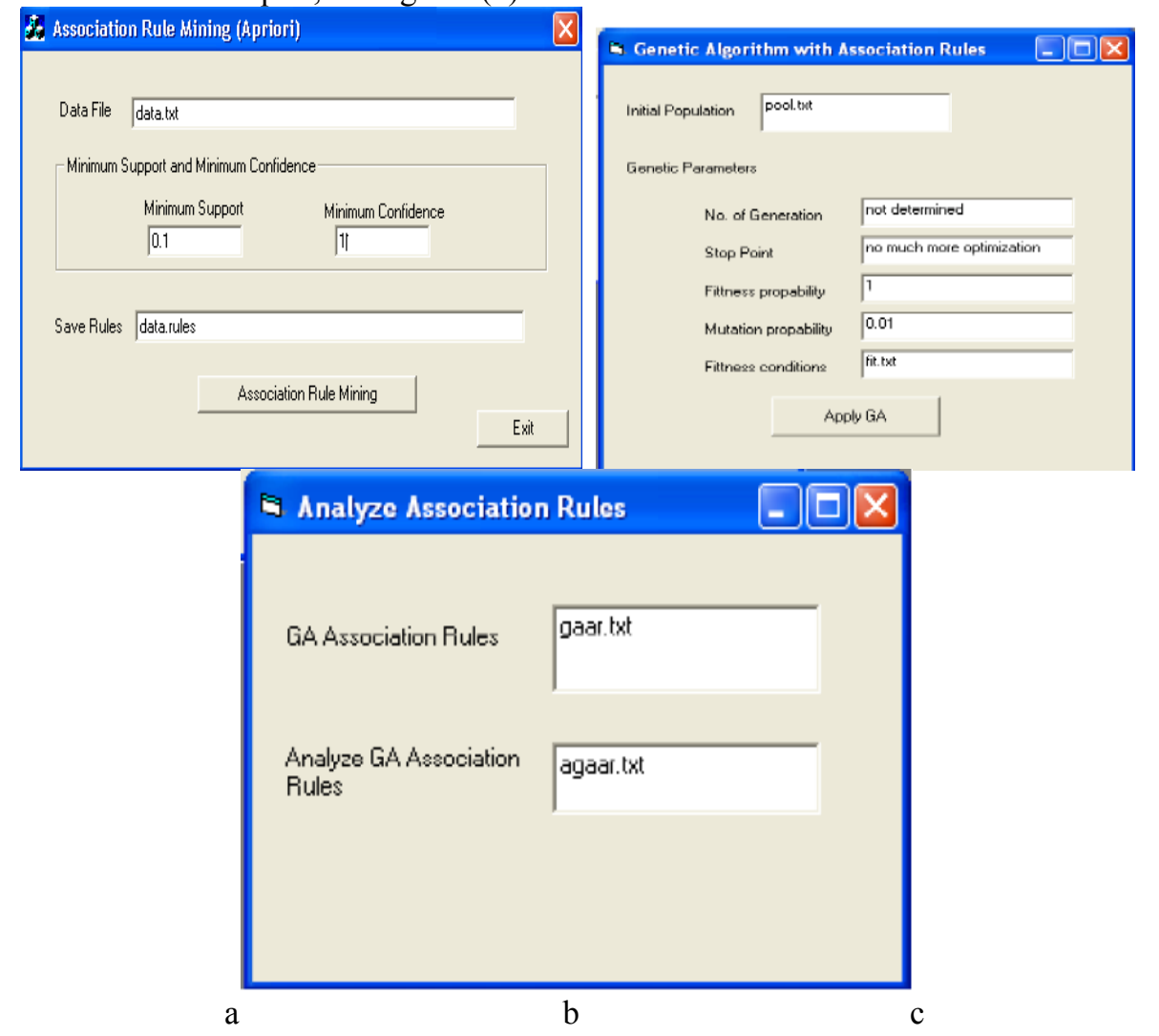

Figure (2): a- applying AR separately on each dimension, b-collect all resulted AR and apply GA, c- analyze resulted patterns from AR-GA with privacy preserving. 
When verify the constructed model analysis and predictions on second part of data we see that, our model advances the following crime analysis:

1. Tactical crime analysis since involves analyzing data to develop information on the where, when, and how of crimes in order to assist officers and investigators in identifying and understanding specific and immediate crime problems.

2. Strategic crime analysis since is concerned with long-range problems and planning for longterm projects. Strategic analysts examine long term increases or decreases in crime, known as crime trends.

3. Administrative crime analysis since focuses on providing summary data, statistics, and general trend information to police managers.

4. Investigative crime analysis since involves profiling suspects and victims for investigators based on analysis of available information.

5. Intelligence analysis since focuses on organized crime, terrorism, and supporting specific investigations with information analysis and presentation.

6. Operations analysis since examines how a law enforcement agency is using its resources. It focuses on such topics as deployment, use of grant funds, redistricting assignments, and budget issues.

Also our model proves and meets the following

- By crime analysis we find meaningful information in vast amounts of data and disseminate this information to officers and investigators in the field to assist in their efforts to apprehend criminals and suppress criminal activity.

- Asses' crime through analysis helps in crime prevention efforts. Preventing crime costs less than trying to apprehend criminals after crimes occur.

- We arrive at the significant attributes for the data mining models. since there are some attributes never appear in prediction and analysis.

- By analyze crimes we could inform law enforcers about general and specific crime trends, patterns, and series in an ongoing, timely manner.

\section{Conclusion and Recommendation}

Our contribution here was to formulate crime pattern detection as machine learning task and to thereby use data mining to support police detectives in solving crimes. We identified all attributes as significant; our modeling technique was able to identify the crime patterns from a large number of crimes making the job for crime detectives easier. We see data mining applied in the context of law enforcement and intelligence analysis holds the promise of alleviating crime related problem. Considering the three dimensions of the crime model make our proposal can help the police to find the criminal quickly by predicting location of the next crime and the residence of the criminal. This paper presents a proposed three correlated dimensional model AR-GA of identifying a criminal, in the absence of witness or any clue by the forensic experts. In these situations, here we have tried to identify the criminal by correlate the criminal with crime and location of the crime using mining and genetic.

The advantages of using KNN imputation in preprocessing are; KNN can predict both qualitative attributes (the most frequent value among the $\mathrm{k}$ nearest neighbors) and quantitative attributes (the mean among the $\mathrm{k}$ nearest neighbors). It can easily treat instances with multiple missing values. It takes in consideration the correlation structure of the data. After extracting the association rules from each dataset separately we tend to mix these associations rules using genetic algorithm. Customizing the genetic algorithm to suite our proposal model, that by propose a schema for encoding the rules, and then making the initial pool is all the extracted rules from the three datasets. Making the crossover point is ----> provide much justify in generating new child rules from parent rules. Fitness function customizes to satisfy the basic conditions in building the association rules according data mining techniques.

Some of the limitations of our proposed model AR-GA include that crime pattern analysis can only help the detective, not replace them. Also data mining is sensitive to quality of input data that may be inaccurate, have missing information, be data entry error prone etc. Also mapping real data to data mining attributes is not always an easy task and often requires skilled data miner and crime data analyst with good domain knowledge. They need to work closely with a detective in the initial phases

\section{References}

[1]. Chen H. , Chung W. , Qin Y., Chau 1., Xu J.ennifer, Wang G., Zheng R., Atabakhsh H., “Crime Data Mining: An Overview and Case Studies", AI Lab, University of Arizona, proceedings National Conference on Digital Government Research, 2003, available at: http://ai.bpa.arizona.edu/

[2]. Fayyad U.M. and Uthurusamy R. ," Evolving data mining into solutions for insights”. Communications of the ACM, 45(8), 28-31, 2002 .

[3]. Chau M., Xu J., and Chen H., "Extracting meaningful entities from police narrative reports". In: Proceedings of the National Conference for Digital Government Research (dg.o 2002), Los Angeles, California, USA. 
[4]. Nath S. V., "Crime Pattern Detection Using Data Mining”, Proceedings of the 2006 IEEE/WIC/ACM international conference on Web Intelligence and Intelligent Agent Technology, 41- 44, 2006.

[5]. Yan J. , Liu N., Yang Q., Zhang B., Cheng Q., Chen Z., "Mining Adaptive Ratio Rules from Distributed Data Sources", Data Mining and Knowledge Discovery, 12, 249-273, 2006, 2005 Springer Science+Business Media, Inc. Manufactured in the United States. [IVSL]

[6]. Malathi. A and. Baboo S. S., "Enhanced Algorithms to Identify Change in Crime Patterns", International Journal of Combinatorial Optimization Problems and Informatics, Vol. 2, No.3, Sep-Dec, 2011, pp. 32-38, ISSN: 2007-1558.

[7]. Mande U., Srinivas Y. and Murthy J.V.R., “An Intelligent Analysis Of Crime Data Using Data Mining \& Auto Correlation Models", International Journal of Engineering Research and Applications (IJERA) ISSN: 2248-9622 www.ijera.com Vol. 2, Issue 4, July-August 2012, pp.149-153 149|P a g e

[8]. Malathi. A, Baboo S. S. and Anbarasi A., “An intelligent Analysis of a City Crime Data Using Data Mining”, 2011 International Conference on Information and Electronics Engineering, IPCSIT vol.6 (2011) C (2011) IACSIT Press, Singapore.

[9]. Sathyaraj S. R., Thangavelu A., Balasubramanian S., Sridhar R., Chandran M. and Prashanthi M. D., "Clustered Spatial Association Rule To Explore Large Volumes Of Georeferenced Crime To Crime Data", 3rd International Conference On Cartography And Gis 15-20 June, 2010, Nessebar, Bulgaria.

[10]. Mande U., Srinivas Y. and Murthy J.V.R., "Criminal Mapping Based On Forensic Evidences Using Generalized Gaussian Mixture Model”, The International Journal of Computer Science \& Applications (TIJCSA), Volume 1, No. 4, June 2012 ISSN - 2278-1080, Available Online at http://www.journalofcomputerscience.com/

[11]. Chen N. and Wang Y., " Prediction of Series Criminals: An Approach Based on Modeling", 2010 International Conference on Computational and Information Sciences.

[12]. Yu C.H., Ward M. W., Morabito M., and Ding W., “ Crime Forecasting Using Data Mining Techniques”, 2011 11th IEEE International Conference on Data Mining Workshops.

[13]. Al-Janabi Kadhim B. S., “ A Proposed Framework for Analyzing Crime Data Set Using Decision Tree and Simple K-Means Mining Algorithms", Journal of Kufa for Mathematics and Computer V o 1. 1, N o. 3, m a y, 2011 , p p .8 - 24 